Open J. Math. Anal., Vol. 2(2018), No. 2, pp. 89 - 92

Website: https://pisrt.org/psr-press/journals/oma/

ISSN: 2616-8111 (Online) 2616-8103 (Print)

http://dx.doi.org/10.30538/psrp-oma2018.0020

\title{
OLD SYMMETRY PROBLEM REVISITED
}

\author{
ALEXANDER G. RAMM ${ }^{1}$
}

\begin{abstract}
It is proved that if the problem $\nabla^{2} u=1$ in $D,\left.u\right|_{S}=0$, $u_{N}=m:=|D| /|S|$ then $D$ is a ball. There were at least two different proofs published of this result. The proof given in this paper is novel and short.
\end{abstract}

Mathematics Subject Classification: 35B06; 35R30; 35J05.

Key words and phrases: Symmetry problems.

\section{Introduction}

Let $D$ be bounded smooth connected domain in $\mathbb{R}^{3}, S$ be its boundary, $N$ is the outer unit normal to $S, u_{N}$ is the normal derivative of $u$ on $S,|D|$ is the volume of $D$ and $|S|$ is the suraface area of $S$. Various symmetry problems were considered in [1, 2].

Consider the problem

$$
\nabla^{2} u=1 \quad \text { in } \quad D,\left.\quad u\right|_{S}=0,\left.\quad u_{N}\right|_{S}=m=|D| /|S| .
$$

Our result is the following:

Theorem 1.1. If problem (1) is solvable then $D$ is a ball.

This result was proved by different methods in [3] and in [4]. The proof, given in the next section, is novel, short and is based on a new idea. We assume that $D \subset \mathbb{R}^{2}$ so that $S$ is a curve. Then the ball is a disc.

Received 14-10-218. Revised 21-11-2018. Accepted 21-11-2018.

1 Corresponding Author

(C) 2018 Alexander G. Ramm. This is an open access article distributed under the Creative Commons Attribution License, which permits unrestricted use, distribution, and reproduction in any medium, provided the original work is properly cited. 


\section{Proof of Theorem 1.1}

Proof. Let $s$ be the curve length, $\mathbf{s}$ be the point on $S$ corresponding to the parameter $s,\{x(s), y(s)\}$ be the parametric representation of $S, \mathbf{s}=x(s) e_{1}+$ $y(s) e_{2}$, where $\left.\left\{e_{j}\right\}\right|_{j=1,2}$ is a Cartesian basis in $\mathbb{R}^{2}$. It is known that $\frac{d \mathbf{s}}{d s}=\mathbf{t}(s)$ is the tangent unit vector to $S$ at the point $\mathbf{s}$ and

$$
\frac{d \mathbf{t}}{d s}=k(s) \nu(\mathbf{s}),
$$

where $k(s) \geq 0$ is the curvature of $S$ and $\nu(\mathbf{s})$ is the normal to $S$. Since $u_{N}=$ $\nabla u \cdot N=m>0$ on $S$ the convexity of $S$ does not change sign, so $\nu$ does not change sign, $k(s)>0 \forall s \in S$ and $N(s)=-\nu(\mathbf{s}) \forall s \in S$. Differentiate the identity $u(x(s), y(s))=0$ with respect to $s$ and get $\nabla u \cdot \mathbf{t}=\mathbf{0}$. Differentiate this identity and use (1)-(2) to get

$$
u_{x x} t_{1}^{2}(s)+2 u_{x y} t_{1}(s) t_{2}(s)+u_{y y} t_{2}^{2}(s)+\nabla u \cdot k(s) \nu(s)=0,
$$

where $\mathbf{t}=t_{1} e_{1}+t_{2} e_{2}$. Rewrite (3) as

$$
u_{x x} t_{1}^{2}(s)+2 u_{x y} t_{1}(s) t_{2}(s)+u_{y y} t_{2}^{2}(s)=m k(s) .
$$

Equation (4) holds in every coordinate system obtained from $\{x, y\}$ by rotations. Clearly $u_{x x}(s), u_{y y}(s), u_{x y}(s)$ cannot vanish simultaneously due to (4). Also $u_{x x}(s), u_{y y}(s)$ cannot vanish simultaneously due to the first equation in (1).

Equation (4) holds in any coordinate system obtained from a fixed Cartesian system by rotations. Equation (1) on the boundary yields:

$$
u_{x x}+u_{y y}=1 .
$$

We prove that (4) and (5) are not compatible (lead to a contradiction) except when $S$ is a circle.

Let $u_{x x}:=p, u_{x y}:=q$. Denote by $A$ the $2 \times 2$ matrix with the elements $A_{11}=p, A_{22}=1-p$, where (5) was used, $A_{12}=A_{21}=q$. Let $I$ be the identity matrix. The equation $\operatorname{det}(A-\lambda I)=\lambda^{2}-\lambda-p^{2}-q^{2}+p=0$ has two solutions, so the eigenvalues of $A$ are:

$$
\lambda_{ \pm}=\frac{1}{2} \pm\left(\frac{1}{4}+p^{2}+q^{2}-p\right)^{1 / 2}=\frac{1}{2} \pm\left[\left(\frac{1}{2}-p\right)^{2}+q^{2}\right]^{1 / 2} .
$$

The corresponding eigenvectors are

$$
e_{1}=\{1, \gamma\}, \quad e_{2}=\{-\gamma, 1\}, \quad \gamma:=\frac{q}{p+\lambda_{+}-1} .
$$

Note that $\lambda_{+}+\lambda_{-}=1, \lambda_{+} \lambda_{-}=-p^{2}-q^{2}+p$. Thus, $\lambda_{+}>0$. The eigenvectors are orthogonal: $e_{1} \cdot e_{2}=0$ but not normalized: $\left\|e_{1}\right\|^{2}=\left\|e_{2}\right\|^{2}=1+\gamma^{2}$. Since $\left\|e_{1}\right\|^{2}$ is invariant under rotations of a Cartesian coordinate system, so is $\gamma^{2}$. Let $w:=\left\{t_{1}, t_{2}\right\}$. Then (4) implies

$$
(A w, w)=m k(s)>0 .
$$


Since $e_{1}$ and $e_{2}$ form an orthogonal basis in $\mathbb{R}^{2}$ one can find unique constants $c_{1}, c_{2}$ such that

$$
c_{1} e_{1}+c_{2} e_{2}=w \text {. }
$$

Solving this linear algebraic system for $c_{1}, c_{2}$ one gets:

$$
c_{1}=\frac{t_{1}+\gamma t_{2}}{\Delta}, \quad c_{2}=\frac{t_{2}-\gamma t_{1}}{\Delta},
$$

where $\Delta=1+\gamma^{2}$ is the determinant of the matrix of the system (99).

Substitute $w$ from (9) into (8) and get:

$$
\left[c_{1}^{2} \lambda_{+}+c_{2}^{2} \lambda_{-}\right]\left(1+\gamma^{2}\right)=m k(s)>0,
$$

where we have used the relations: $A e_{j}=\lambda_{j} e_{j}, \lambda_{1}:=\lambda_{+}, \lambda_{2}:=\lambda_{-},\left(e_{1}, e_{2}\right)=0$, $\left\|e_{j}\right\|^{2}=1+\gamma^{2},\left(A e_{j}, e_{j}\right)=\lambda_{j}\left(1+\gamma^{2}\right), j=1,2$. Using (10) one gets from (11):

$$
\left(t_{1}+\gamma t_{2}\right)^{2} \lambda_{+}+\left(t_{2}-\gamma t_{1}\right)^{2} \lambda_{-}=m k(s)\left(1+\gamma^{2}\right)>0 .
$$

We prove that 12 leads to a contradiction unless $S$ is a circle.

Assume first that $\lambda_{-}<0$ and recall that $\lambda_{+}>0$. Choose a point $s \in S$ and the Cartesian coordinate system such that $t_{1}(s)+\gamma(s) t_{2}(s)=0$. This is possible since $\gamma^{2}$ is invariant under rotations and the only restriction on the real-valued $t_{1}, t_{2}$ is the relation $t_{1}^{2}+t_{2}^{2}=1$. Since $\lambda_{-}<0$ and $t_{2}-\gamma t_{1} \neq 0$, we have a contradiction with inequality (12).

Assume now that $\lambda_{-} \geq 0$ and $\lambda_{-} \neq \lambda_{+}$. Then the left side of 12 is not a constant as a function of $\left\{t_{1}, t_{2}\right\}$, that is, not a constant with respect to rotations of the coordinate system, while its right side is a constant. Thus, we have a contradiction.

Suppose finally that $\lambda_{-}=\lambda_{+}$. Then $\lambda_{-}=\lambda_{+}=\frac{1}{2}$ at any $s \in S$. This implies by formula (6) that $p=\frac{1}{2}, u_{y y}=\frac{1}{2}$ and $q=0$ on $S$ for all $s \in S$. By formula (7) one gets $\gamma=0,\left\|e_{j}\right\|=1$. Consequently, by formula (4), it follows that $\kappa(s)=\frac{1}{2 m}$. Thus, the curvature of $S$ is a constant, so $S$ is a circle of a radius $a$. Thus, $m=\frac{\pi a^{2}}{2 \pi a}=\frac{a}{2}, k(s)=\frac{1}{a}$ and the solution to problem (1) is $u=\frac{|x|^{2}-a^{2}}{4}$. Obviously this $u$ solves equation (1) and satisfies the first boundary condition in (1). The second boundary condition is also satisfied: $\left.u_{N}\right|_{S}=a / 2$.

Theorem 1.1 is proved in the two-dimensional case. We leave to the reader to consider the three-dimensional case, see [5]. Theorem 1.1 is proved.

\section{Competing Interests}

The author declares that he has no competing interests.

\section{REFERENCES}

1. Ramm, A. G. (2005). Inverse problems: mathematical and analytical techniques with applications to engineering. Springer.

2. Ramm, A. G. (2017). Scattering by obstacles and potentials. World Scientific Publishers, Singapore.

3. Ramm, A. G. (2013). Symmetry problem. Proceedings of the American Mathematical Society, 141(2), 515-521. 
4. Serrin, J. (1971). A symmetry problem in potential theory. Archive for Rational Mechanics and Analysis, 43(4), 304-318.

5. Ramm, A. G. (2018). Necessary and sufficient condition for a surface to be a sphere. Open J. Math. Anal., 2(2), 51-52.

\section{Alexander G. Ramm}

Department of Mathematics, Kansas State University, Manhattan, KS 66506, USA.

e-mail: ramm@math.ksu.edu 